\title{
Rate effects in a proportional counter with resistive cathode
}

\author{
Carmen C. Bueno ${ }^{\mathrm{a}, \mathrm{b}}$, M.M. Fraga ${ }^{\mathrm{c}, *}$, Josemary A.C. Gonçalves $^{\mathrm{a}, \mathrm{b}}$, R. Ferreira Marques ${ }^{\mathrm{c}}$, \\ A.J.P.L. Policarpo ${ }^{\mathrm{c}}$, M. Damy de S. Santos ${ }^{\mathrm{a}, \mathrm{b}}$ \\ ${ }^{a}$ Instituto de Pesquisas Energéticas e Nucleares, Comissão Nacional de Energia Nuclear 05508-900, Caixa Postal 11049, São Paulo, Brazil \\ ${ }^{\mathrm{b}}$ Departamento de Física, Pontificia Universidade Católica de São Paulo 01303-050, São Paulo, Brazil \\ ${ }^{\mathrm{c}}$ Laboratório de Instrumentação e Física Experimental de Partículas, LIP - Coimbra, Departamento de Física, Universidade de Coimbra, \\ 3000 Coimbra, Portugal
}

Received 27 May 1997; received in revised form 16 December 1997

\begin{abstract}
In this work, the rate effects associated with the gain of detectors with resistive electrodes are considered. To study these effects it was decided to use the classical proportional counter geometry, with a cylindrical glass tube as the cathode, rather than parallel plate devices. Indeed, the generality of the information obtained is not affected and the cylindrical proportional counter geometry has several advantages to gather detailed experimental data and to compare it with simple electric field calculations. A self-consistent set of data is presented for the effects of counting rate from a few tens of $\mathrm{Hz}$ up to several hundreds of $\mathrm{Hz}$, using several gas mixtures, different anode wires, cathode radii and thickness of the resistive electrodes. Although a few sources of systematic errors can be identified, all the observed experimental data can be quantitatively explained. Indeed, the measured gas amplification, from a few tens up to $\sim 5 \times 10^{4}$ and for the range of counting rates quoted above, depends only on the local electric field as it should be expected. These results are directly relevant to approach the problem of counting efficiency in resistive plate chambers. (C) 1998 Elsevier Science B.V. All rights reserved.
\end{abstract}

PACS: $29.40 . \mathrm{Cs} ; 29.40 . \mathrm{Gx}$

Keywords: Proportional counters; Gaseous detectors; Resistive cathodes

\section{Introduction}

Detectors using highly resistive electrodes have been used for many years. The more popular ones are the Pestov counters [1,2] and the Resistive Plate Chambers, RPC, introduced by Santonico

\footnotetext{
*Corresponding author. E-mail: margarida@filip3.fis.uc.pt.
}

and Cardarelli in 1981 [3]. Lately, RPCs have been studied extensively, specially since they were considered as suitable detectors for the construction of a first level muon trigger at the upcoming CERN's Large Hadron Collider (LHC). Indeed, they feature very large areas with low cost, simplicity of operation and good time resolution.

The more recent work with these detectors concerns the study of counting rate effects and their operation in the proportional and streamer regions, 
the research on the effect of high concentrations of UV absorbing gases and the use of narrow and wide size gaps (see Refs. [4-7]). The fact that RPCs are essentially spark-protected Parallel Plate Chambers (PPC) implies also the interest of research in the direction of good position resolution [8]. In order to take advantage of this and other unique properties of RPCs, in particular, at the demanding LHC environment [8,9], systematic studies of the factors affecting counting efficiency had to be undertaken [6]. In this work we consider mainly the rate effects associated with the gain of the detectors with resistive electrodes. These effects will depend on the nature of the gas mixture and on the electric field (the physical processes associated with the gas amplification mechanisms are essentially a consequence of these parameters). The field characteristics will in turn be determined by the geometry of the detector, the electric properties of its electrodes and the current through them. To study the rate effects associated with resistive electrodes, it was decided to use the classical proportional counter geometry, with a resistive cylindrical tube as the cathode, rather than the parallel plate geometry. The generality of the information obtained is not affected by the geometry. The cylindrical proportional counter is actually profitable, as it provides good energy resolution and, therefore, the detection and measurement of small variations of gain and current, and a very good definition of its regime of operation (proportional, saturated or streamer mode). The exact regime of operation of RPCs bombarded with minimum ionising particles (MIPs) or $\gamma$-rays can be difficult to assess, as well as the eventual existence of spacecharge effects. On the other hand, the symmetry of the cylindrical device allows the calculation of the electric field in a way that is essentially as simple as the parallel plate. This is an important consideration in this work, as a quantitative and systematic approach to the problem of rate effect is undertaken, using detailed experimental data.

\section{Experimental apparatus and methods}

Cylindrical detectors, with metallic anode wires stretched along their axes and cathode body made out of glass have been studied. The dimensions of the two samples investigated, called A and B, are given in Table 1.

Detectors A and B were divided into two sections (see Fig. 1). One-half was covered internally with a thin grounded layer of aquadag (detectors $A_{i}$ and $B_{i}$ ), hereafter internal cathode tubes, which allows the tube to work like a conventional proportional counter, while the other half was covered externally by the same layer (detectors $A_{e}$ and $B_{e}$ ), hereafter external cathode tubes. Both-tubes were irradiated with $22 \mathrm{keV} \mathrm{X}$-rays from a ${ }^{109} \mathrm{Cd}$ source. Different counting rates were obtained by interposing, between the source and the tube, $\mathrm{Pb}$ foils with holes of different diameters. However, since the rate effect depends on the number of electron avalanche per second per unit length of the anode wire, it will be desirable to carry out the experiment by changing the X-ray intensity under a fixed diameter of the hole of the $\mathrm{Pb}$ foil.

The gas mixtures were prepared passing the gas components through calibrated flowmeters and were admitted into the chambers in a continuous flow regime, at atmospheric pressure. The gases used were the commercially available methane 99.995\% and pre-mixed argon/methane $90: 10$ (P10) $(>99.98 \%)$. The measurements were performed at room temperature $(\sim 293 \mathrm{~K})$.

Table 1

Dimension of the two glass cylindrical tubes and anode wires

\begin{tabular}{|c|c|c|c|c|}
\hline Detector & Length (mm) & $\begin{array}{l}\text { Internal diameter } \\
(\mathrm{mm})\end{array}$ & $\begin{array}{l}\text { Thickness } t(\mathrm{~mm}) \text { of } \\
\text { the glass cathodes }\end{array}$ & Anode wire \\
\hline A & 200 & 13.9 & 0.7 & Tungsten, $127 \mu \mathrm{m} \phi$ \\
\hline $\mathrm{B}$ & 200 & 12.7 & 1.1 & $\begin{array}{l}\text { Tungsten, } 127 \mu \mathrm{m} \phi \\
\text { Stainless steel, } 50 \mu \mathrm{m} \phi\end{array}$ \\
\hline
\end{tabular}




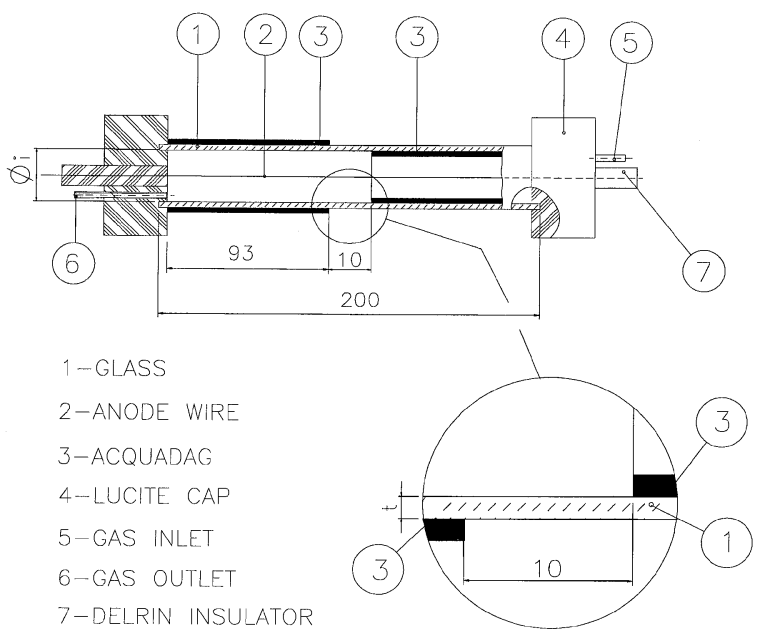

Fig. 1. Schematic drawing of the resistive cathode proportional counter.

The anode signals were fed to a charge-sensitive preamplifier and a linear amplifier with a time constant of $2 \mu \mathrm{s}$. The output of the amplifier was connected to a multichannel analyser to measure the collected charge. An adequate charge calibration was performed, injecting a known amount of charge at the input of the preamplifier with a pulse generator and a $2 \mathrm{pF}$ test capacitance.

The applied voltages were controlled to within $\pm 1 \mathrm{~V}$ and kept within the proportional region.

\section{Model}

For measuring the characteristic dependence of the current flowing through the glass on the voltage, the internal and external surfaces of one of the tubes were covered with conductive paint, a voltage was applied between them and the current measured with a picoammeter. The glass was found to obey Ohm's law and the value measured for its resistivity, $\rho$, was $1.2 \times 10^{13} \Omega \mathrm{cm}$.

In the absence of radiation, when the voltage is applied to the anode wire, a potential difference is established between the internal and the external surfaces of the glass tube, due to the polarisation of the glass (specific dielectric constant $k=5$ ). But due to the finite resistivity of this material, a current will flow between the two surfaces until the internal and the external surfaces of the tube are at the same potential. When the tube is irradiated, the internal surface of the tube begins to charge up and a current flows through the glass wall. When equilibrium is reached, the current in the glass equals the current in the gas, and a potential difference is established between the internal and external surfaces. Assuming infinite wire length and uniform irradiation of the detector, the electric field in the gas region is then given by

$E(r)=\frac{\left(V_{0}-I_{0} R\right)}{\ln \left(r_{c} / r_{a}\right)} \frac{1}{r}$

where $V_{0}$ is the applied voltage, $I_{0}$ the current due to irradiation of the detector, $r_{\mathrm{c}}$ the inner radius of the tube, $r_{\mathrm{a}}$ the anode radius and $R$ is the resistance of the irradiated region of the tube. If the surface currents are neglected, this is given by

$R=\frac{\rho}{2 \pi h} \ln \frac{r_{b}}{r_{c}}$

where $\rho, r_{\mathrm{b}}$ and $h$ are the resistivity of the glass (assumed to be uniform), the external radius of the tube and the axial length of the irradiated region (i.e., the effective counter length), respectively. The assumptions referred to previously were approximated, in particular making $h \gg r_{c}$. E(r) tends to the expression of the electric field inside a conventional cylindrical counter when $I_{0}$ is negligibly small.

\section{Experimental results and discussion}

Concerning the gas supply, detectors A and B, equipped with tungsten wires of $127 \mu \mathrm{m}$ diameter, were connected in series such that the gas mixture, P10, flows from A to B. For each of them, charge gains were measured for both the internal and external cathode sections, at the same applied voltages. These gains are represented as a function of the applied voltage (Fig. 2a) and as a function of the reduced electric field at the anode surface $\left(E_{\mathrm{a}} / P\right.$, $P$ being the pressure) (Fig. 2b), for a counting rate of about $70 \mathrm{~Hz}$. The conventional expression for $E_{\mathrm{a}}$ in a cylindrical counter was used, i.e., $I_{0}$ was put 

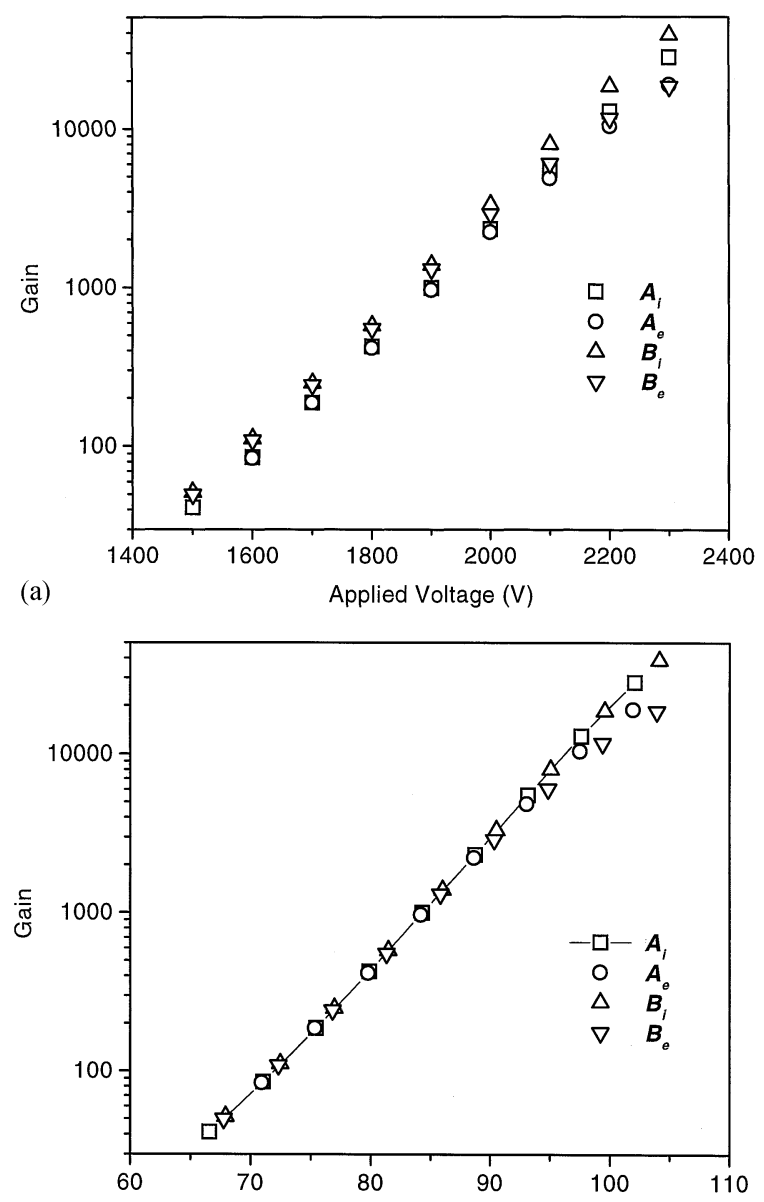

(b)

$\mathrm{E}_{\mathrm{a}} / \mathrm{P}(\mathrm{V} / \mathrm{cm}$.torr $)$

Fig. 2. Measured gains in the P-10 mixture, for detectors A and $\mathrm{B}$ (for both internal and external cathode sections), as a function of: (a) the applied voltage; (b) the reduced electric field at the anode computed for the internal cathode detectors.

equal to zero in expression (1). The analysis of these curves shows that

1. the difference in gains for detectors $A_{i}$ and $B_{i}$ is only due to the different electric field configuration in each case (see Table 1);

2. for reduced electric fields smaller than $87 \mathrm{~V} / \mathrm{cm}$ Torr, the gains are all approximately equal; however, above this value there is a clear decrease of the gains measured in the regions of the tubes with external cathodes, the decrease being more pronounced for detector $\mathrm{B}$, which has a thicker glass wall. It is also clear that the gain drop is more important for increasing applied voltages.

No rate effects should be associated with the internal cathode detector for the moderate counting rates, $v$, and X-ray flux densities used. Nevertheless, the dependence of the gain on $v$ was investigated, for both the internal and external cathode tubes:

1. Detectors $\mathrm{A}_{\mathrm{i}}$ and $\mathrm{B}_{\mathrm{i}}$ : as shown in Fig. 3, the ratio, $G_{\mathrm{iL}} / G_{\mathrm{iH}}$, of the gains measured at the lowest and at the highest counting rates (70 and $510 \mathrm{~Hz}$, respectively) is approximately unity, along the whole range of applied voltages, showing that space-charge effects $[10,11]$ are absent (see Fig. 3). It was then confirmed that the gain of the internal cathode detectors does not depend on the counting rate, up to the highest that was used.

2. Detectors $A_{e}$ and $B_{e}$ : there is a decrease of the gain ratio with increasing counting rate, the effect being more pronounced for higher applied voltages. This effect can be seen in Fig. $4 \mathrm{a}$ and $4 \mathrm{~b}$, where the ratios $G_{\mathrm{e}} / G_{\mathrm{i}}$ for different counting rates are plotted as a function of the applied voltages for the detectors $\mathrm{A}_{\mathrm{e}}$ and $\mathrm{B}_{\mathrm{e}}$, respectively.

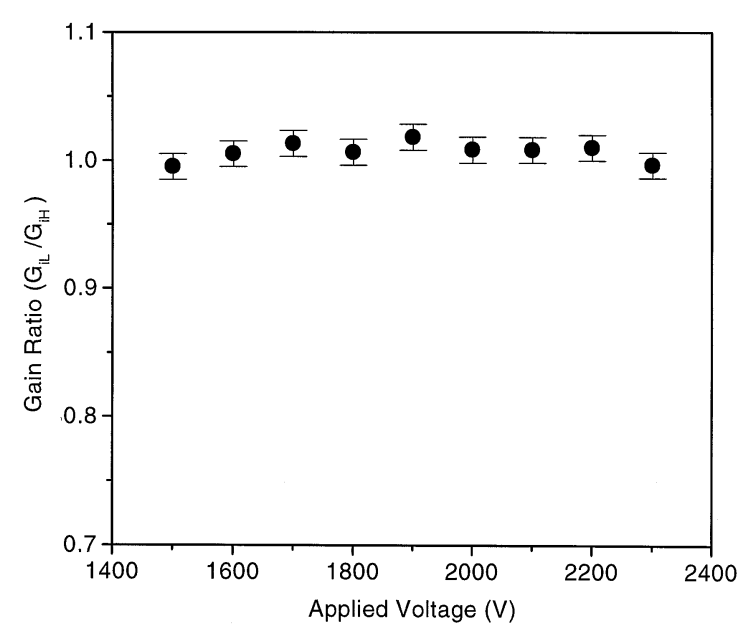

Fig. 3. Ratio $\left(G_{\mathrm{iL}} / G_{\mathrm{iH}}\right)$ between the gains measured in the P-10 mixture for detector $\mathrm{A}_{\mathrm{i}}$ (internal cathode) at the lowest and the highest counting rates, 70 and $510 \mathrm{~Hz}$, respectively, as a function of the applied voltage. 

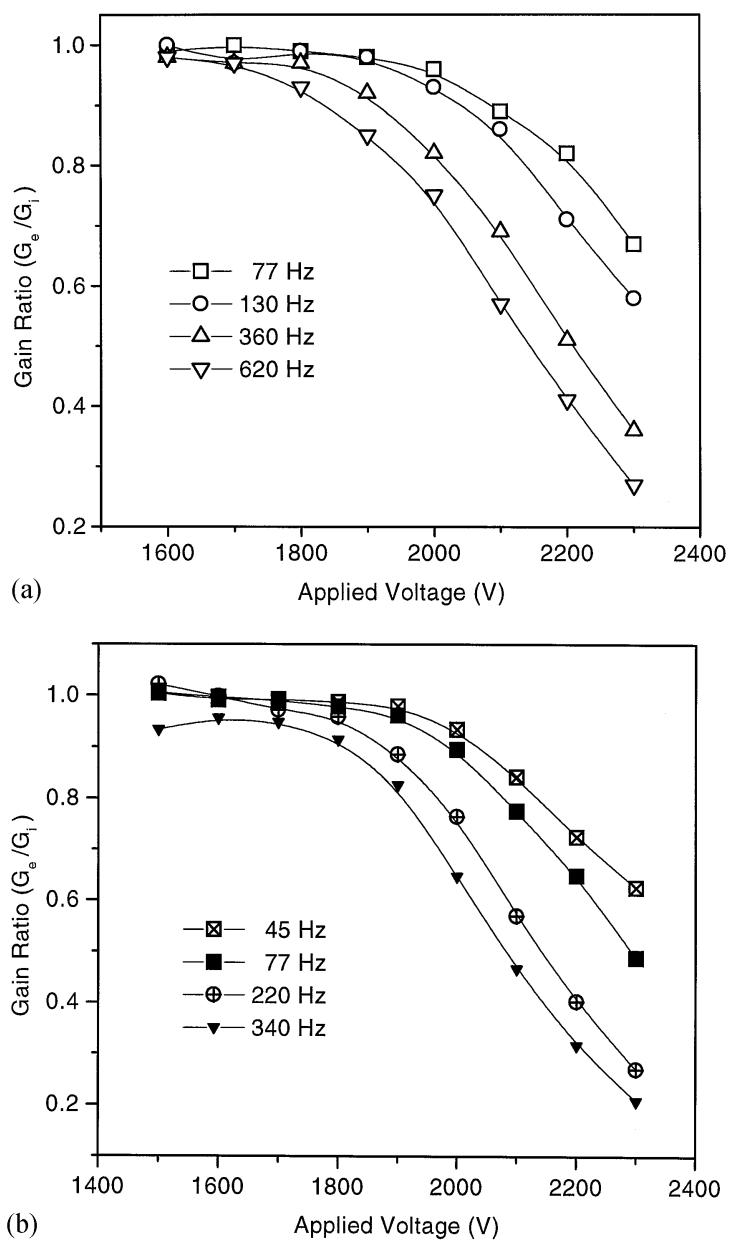

Fig. 4. Ratios between the gains measured, at the same applied voltage, with the P-10 mixture for the external and the internal cathode region of the detector $\left(G_{\mathrm{e}} / G_{\mathrm{i}}\right)$ as a function of the applied voltage, for several counting rates: (a) detector $\mathrm{A}$; (b) detector $\mathrm{B}$.

$G_{\mathrm{e}}$ and $G_{\mathrm{i}}$ are the gains measured, for the same applied voltage and a similar counting rate, with the external and the internal cathode tubes, respectively.

According to the model established above, the gain drop in the external cathode tubes does not depend separately on the applied voltage or the counting rate, but rather on the product $I_{0}=Q v$, $Q$ being the total charge developed per avalanche [12]. In fact, if we plot the ratios $G_{\mathrm{e}} / G_{\mathrm{i}}$ represented in Fig. 4, now as a function of the current $I_{0}$, they clearly overlap along two curves, one for each tube (see Fig. 5). It is apparent that, due to its higher resistance, the gain in detector $B_{e}$ starts to decrease for lower currents than in detector $A_{e}$.

The same experimental procedure was repeated for detector B equipped with a $50 \mu \mathrm{m} \phi$ stainless steel wire and the results are plotted in Fig. 6, again showing that the gain decrease is determined only by the current.

The dependence on the gas mixture was also investigated by comparing the gain drops in the P-10, P-70 (Ar/CH $\left./ \mathrm{CH}_{4}, 30: 70\right)$ and P-82 $\left(\mathrm{Ar} / \mathrm{CH}_{4}\right.$, $18: 82$ ) mixtures (see Fig. 7).

Once we know the current $I_{0}$ in the detector $\left(\mathrm{A}_{\mathrm{e}}\right.$ or $\mathrm{B}_{\mathrm{e}}$ ) for a given applied voltage and the resistance $R$ (Eq. (2)), we can calculate the corresponding $E_{\mathrm{a}} / P$ value (Eq. (1)), the real reduced electric field, and determine, from the experimental data, the value of the gain for the same $E_{\mathrm{a}} / P$ value, in the internal cathode detector. The ratios $G_{\mathrm{e}} / G_{\mathrm{i}}$ should then be equal to unity for any geometry, rate, voltage and nature of the mixture. In Fig. 8 we represent the ratios $G_{\mathrm{e}} / G_{\mathrm{i}}$ as a function of $E_{\mathrm{a}} / P$, for detectors $\mathrm{A}$ and $\mathrm{B}$, for the counting rates used. Since the value of the irradiation length is not well known it was left as a free parameter. The resulting value, $h=16$ and $13.5 \mathrm{~mm}$ for detectors $\mathrm{A}$ and $\mathrm{B}$, respectively, is consistent with the geometry of

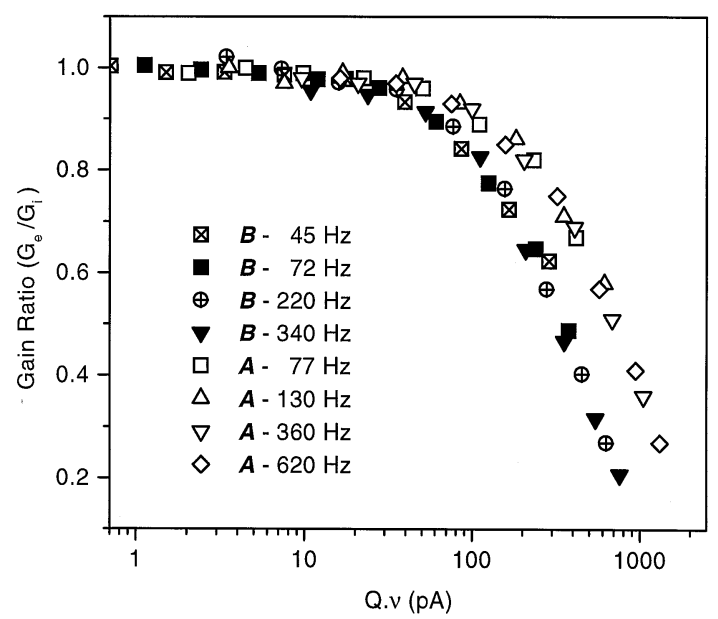

Fig. 5. Comparison of the variation of the relative gains $\left(G_{\mathrm{e}} / G_{\mathrm{i}}\right)$ shown in Fig. $4 \mathrm{a}$ and $4 \mathrm{~b}$, as a function of the current produced in the external cathode detector by the ionizing radiation. 


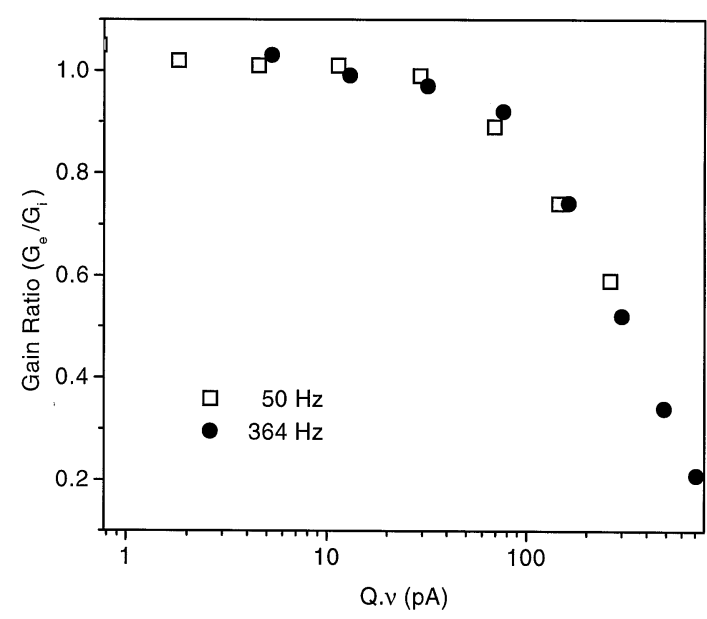

Fig. 6. Ratios $G_{\mathrm{e}} / G_{\mathrm{i}}$ for the P-10 mixture as a function of the current produced in the external cathode section of detector B (equipped with a $50 \mu \mathrm{m} \phi$ stainless steel wire) by the ionizing radiation, for two counting rates.

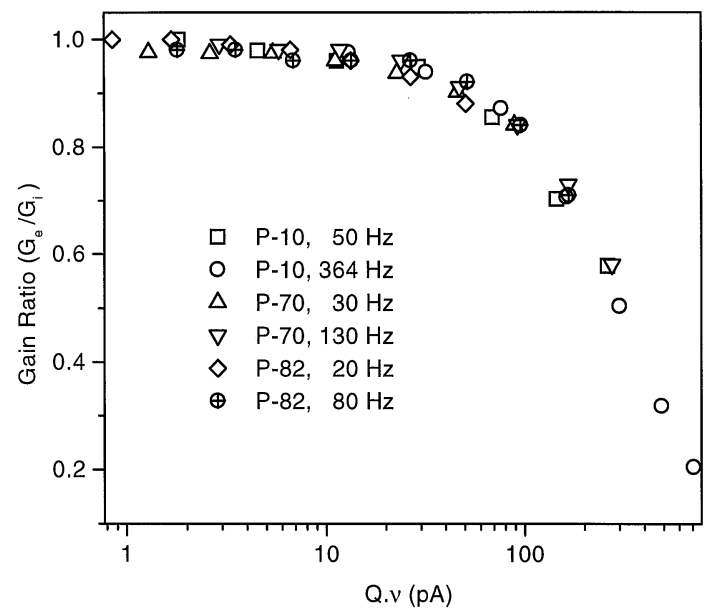

Fig. 7. Comparison of the variation of the relative gains $\left(G_{\mathrm{e}} / G_{\mathrm{i}}\right)$ for three different argon/methane mixtures as a function of the current produced in the external cathode section of detector B, by the ionizing radiation.

irradiation. The errors associated to the ratios $G_{\mathrm{e}} / G_{\mathrm{i}}$ are essentially due to uncertainties in the charge measurements and calibration procedure and in the determination (by polynomial interpolation) of the gain in the internal cathode detector (of the same geometry).
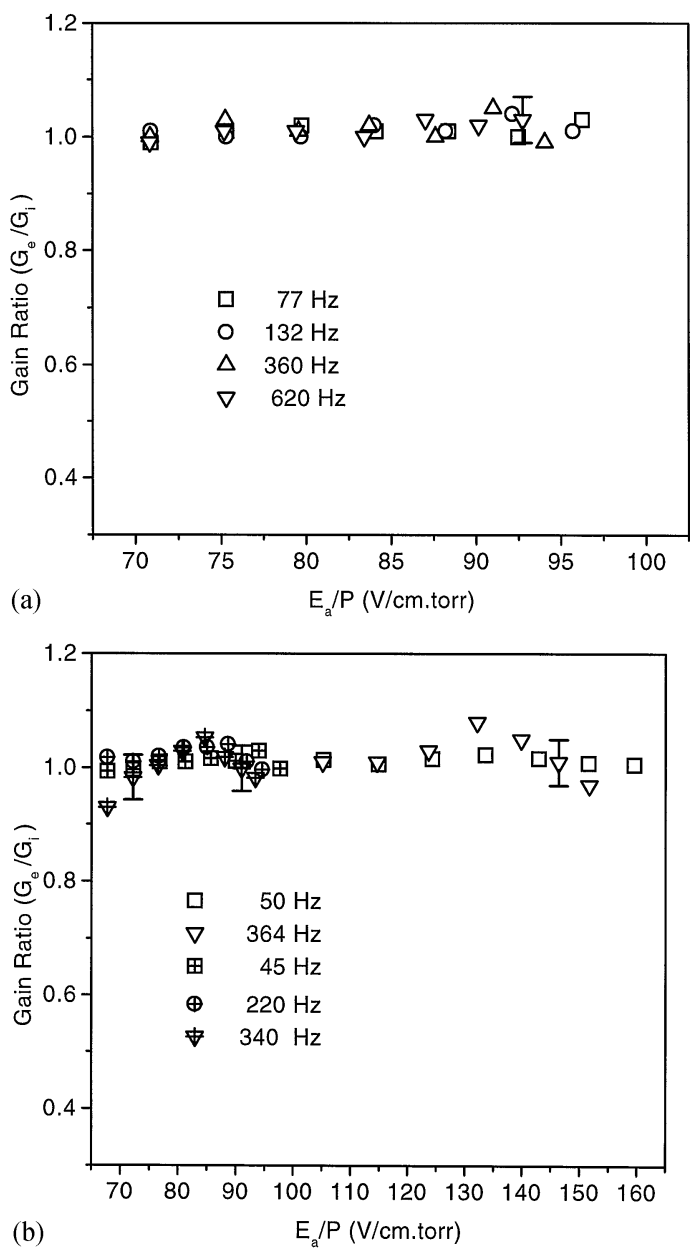

Fig. 8. Ratio between the gain measured for the P-10 mixture, with the external cathode detector and the value of the gain for the same $E_{\mathrm{a}} / P$ value, in the internal cathode detector, as a function of $E_{\mathrm{a}} / P$, for various counting rates. (a) detector A; (b) detector B. Open symbols, $\phi_{\mathrm{a}}=50 \mu \mathrm{m} ;+$ centred symbols, $\phi_{\mathrm{a}}=$ $127 \mu \mathrm{m}$ (the data corresponding to $v=77 \mathrm{~Hz}$ are absent from this figure to avoid a high density of experimental points for the lower $E_{\mathrm{a}} / P$ values).

\section{Conclusions}

A self-consistent set of data is presented for rate effects, from a few tens of $\mathrm{Hz}$ up to several hundreds of $\mathrm{Hz}$, associated to resistive detectors, cylindrical glass proportional counters $\left(\rho \sim 10^{13} \Omega \mathrm{cm}\right)$. Several geometries and mixtures were tested, using different anode wires, cathode radii and thickness of the glass resistive electrodes. 
Preliminary observations indicate that the relaxation times associated with the detectors are of the order of tens of seconds and, thus, for the rates used, a simple model that assumes an equilibrium state could be established. Experimental conditions were such that a simple quantitative approach can be used to calculate the electric field in the region of gas amplification, taking into account the geometry, electrical properties of the electrodes and current in the detector due to the ionizing radiation. Although several sources of systematic errors can be identified (mainly, the exact length of the irradiated region, the resistivity of the glass and its uniformity and the charge calibration), all the observed experimental data can be quantitatively explained. Indeed, the measured gas amplification, from a few tens up to $\sim 5 \times 10^{4}$, depends only on the local electric field, as it should be expected. This conclusion naturally applies also to any other type of geometry, in particular to RPCs, for which the same simple quantitative approach can be used, as the decrease of their counting efficiency with counting rate is directly associated to the gain drop.

\section{Acknowledgements}

This work was supported in Portugal by JNICT (contract CERN/CA/1102/96), and in Brasil by FAPESP and CNPq.

\section{References}

[1] W.B. Atwood et al., Nucl. Instr. and Meth. 206 (1983) 99.

[2] A.R. Frolov et al., Nucl. Instr. and Meth. A 307 (1991) 497.

[3] R. Santonico, R. Cardarelli, Nucl. Instr. and Meth. 187 (1981) 377.

[4] C. Bacci et al., Nucl. Instr. and Meth. A 352 (1995) 552.

[5] I. Duerdoth et al., Nucl. Instr. and Meth. A 348 (1994) 303.

[6] E. Cerron Zaballos et al., Nucl. Instr. and Meth. A 367 (1995) 388.

[7] E. Cerron Zaballos et al., Nucl. Instr. and Meth. A 373 (1996) 35.

[8] E. Cerron Zaballos et al., Preprint LIP96/10, Nucl. Instr. and Meth., to be published.

[9] A. Peisert et al., Nucl. Instr. and Meth. A 247 (1986) 453.

[10] R.W. Hendricks, Rev. Sci. Instr. 40 (1969) 1216.

[11] H. Sipilä, V. Vanha-Honko, Nucl. Instr. and Meth. 153 (1978) 461.

[12] R. Bellazzini et al., Nucl. Instr. and Meth. A 247 (1986) 445. 